\title{
Low power GPS drifters with local storage and GSM modem made from off the shelf components
}

\author{
Rolf Hut $^{1}$, Thanda Thatoe Nwe Win ${ }^{2}$, and Thom Bogaard ${ }^{2}$ \\ ${ }^{1}$ Delft University of Technology, Faculty of Civil Engineering and Geosciences, chair of Water \\ Resources Engineering \\ ${ }^{2}$ Delft University of Technology, Faculty of Civil Engineering and Geosciences, chair of Hydrology \\ Correspondence to: Rolf Hut (r.w.hut@tudelft.nl)
}

Abstract. Drifters that track their position are important tools in studying the hydrodynamic behaviour of rivers. Drifters that can be tracked in real time have so far been rather expensive. Recently both GPS receivers and GSM modems have become available at lower prices to tinkering scientists due to the rise of the Open Hardware revolution and the associated Arduino ecosystem. This article serves two goals. Firstly, we provide detailed instructions on how to build a Low Power GPS drifter with local storage and GSM model that we tested in a fieldwork on the confluence of the Chindwin and Ayeyarwady rivers in Myanmar. These instructions allow fellow geoscientists to recreate the device. Secondly, we set the question: "Has the Open Hardware revolution progressed to the point that a low power GPS drifter that wireless transmits its position can be made from Open Hardware component by geoscientists without extensive training or expertise in electrical and software engineering? We feel this question is relevant and timely as more low-cost Open Hardware devices are promoted but in practice applicability often is restricted to the 'tinkering engineer'. We argue that because of the plug and play nature of the components geoscientist should be able to construct these type of devices. However, to get such devices to operate at low power levels that fieldwork often requires detailed (mircro)electrical expertise.

\section{Introduction}

Drifters, devices that naturally float and follow the surface flow lines in a moving water body, are a method to measure important parameters such as surface water velocity distributions, breakthrough curves and mixing parameters. In drifter research it is important to have as many drifters as possible to optimally measure flow patterns (Tinka et al., 2010). Drifters measure their location, almost exclusively using GPS technology and store it on the device, or transmit it using some network 
connection. Currently drifters are made for specific research questions and are either expensive to purchase or are build in house, requiring detailed knowledge of electrical engineering and embedded software (Network, 2012; Tinka et al., 2013), two skills not generally present in geoscientists. In general drifters consist of a location device, often GPS, a logger to store the data and/or a communication unit. Recent developments, most notably the rise of the "maker movement" (Chris, 2012) and its ecosystem based on Arduino, Raspberry Pi and other Open Hardware development boards (Banzi and Shiloh, 2014; Foundation, 2018), have made the individual components of a GPS drifter commercially available as plug and play components for hobbyist and developers. This has led scientists to build GPS loggers geared to their own research need at lower costs. Daniel K. and Peter J. (2012) gave an early overview of the possibilities of the Arduino ecosystem as a low cost alternative for expensive scientific instrumentation. Wickert (2014) developed the Alog, a low power Arduino inspired logger system specifically designed with geoscientific fieldwork in mind.

Relating to using Open Hardware to track GPS positions, Cain and Cross (2018) made a low power GPS logger to track Eastern box turtles. Their device stores the data in the local memory of the Arduino microcontroller, requiring retrieval after the experiment and no real time view on where the turtles are. Pham et al. (2013) and Costanzo (2013) both connected a GPS receiver and a GSM modem to an Arduino to track locations of vehicles. In vehicle tracking usually sufficient power is available through the battery of the car. Lockridge et al. (2016) build a drifter to be used in near shore environments that logged GPS positions locally on an SD card. Trevathan et al. (2012) custom build a suite of near shore bouys that together act as a Wireless Sensor Network (WSN). Finally Tinka et al. (2013) gives a complete overview of all work on drifters (not focussing on low cost) in their introduction and subsequently present their high end, custom designed Floating Sensor Network.

For fieldwork on the Ayeyarwady and Chindwin rivers in Myanmar we were in need of a low cost, low power GPS drifter that stores its data locally and at the same time transmits its location in real time for retrieval of the device. All of the projects cited above, while build using Open Hardware low cost components, are either expensive to purchase or required extensive engineering. In this article we set out to test the question: "Has the Open Hardware revolution progressed to the point that a low power GPS drifter that wireless transmits its position can be made from Open Hardware component by geoscientists without extensive training or expertise in electrical and software engineering?"

This article will explain how we build such a device, details the software designed and present the raw measurements results from the fieldwork on the Irrawaddy river. The detailed analyses of the data falls beyond the scope of this article, which focuses on the development of the data collection devices. We conclude by reflecting on the primary research question about the "build-ability" of our device by geoscientists in the discussion and conclusions. 


\section{2 materials}

The drifter design can be separated in two parts. Firstly the drifter itself, ie. the material that makes the drifter float optimally given the local flow conditions of the field site. Secondly the electronics that do the GPS localization and communication. We describe the electronics first and the drifter design afterwards.

\subsection{Electronic Hardware}

The design criteria for our device were to develop a system that could:

- Track its own location using GPS.

- Upload its location to the internet to both save the measurement data and to be able to locate the device in real time during the measurement campaign.

- store the locations locally in case of no connection to the internet.

- operate for at least two days continuously without losing power.

We decided to buy a ready made solution for each design criteria and connect those together. The electronics that matched our design criteria and we choose are:

- a Particle Electron. A development board with build in GSM modem. The electron comes with a data subscription with near global coverage and an online cloud back-end. Users can program the electron with their own code using an online development environment (Particle, 2018) in the $\mathrm{C}++$ dialect that is also used for the Arduino ecosystem (Arduino, 2018).

- a Particle Asset Tracker Shield, an extension to the Particle Electron, that includes a GPS receiver and an accelerometer. The Particle Electron connects to this shield using a set of headers on the shield. Communication between the Electron and the GPS receiver is done over UART Serial communication using the Tx and Rx pins of the Electron.

- a Sparkfun OpenLog. A board that records any data send to it using UART serial communication onto an SD card.

80

- an $8 \times 12$ centimeter solar panel.

Table 5 list online locations where the components were purchased and prices at the time of writing. All datasheets with detailed technical specifications of the components are available as supplementary material to this article. Connection of the components to each other is straightforward and given in table 5 . 


\subsection{Software and online back-end}

The software required to operate the GPS drifters is divided into two parts: the software that runs on the Particle Electron and the software that runs online. All code used is available on Github and has been Archived on Zenodo (Hut, 2018). This separation between online and firmware was made to facilitate re-use by researchers that want to build on one, but not both, of these parts.

Just as with its spiritual predecessor the Arduino, the Particle Electron stores a single program in its program memory and this program is executed continuously as soon as the Particle Electron is powered. The Particle Electrons is programmed in the same $\mathrm{C}++$ dialect as is used for the Arduino. Ready made examples exist that let the Particle Electron and the Asset Tracker Shield upload GPS locations on a regular bases to the Particle Cloud service. However, these programs keep the cellular connection open continuously which drains too much power for our application. The code was heavily modified to optimize for minimal power consumption. Figure 5 shows the architecture of the code that runs on the Particle Electron. Every minute the device wakes from a low power deep sleep mode and records the GPS coordinates from the Asset Tracker Shield. This is stored both in the volatile memory of the device as well as on the SD card in the OpenLog. Once every fifteen minutes the device will try to connect to the GSM network. If successful, it will upload the 15 latest GPS measurements to the Particle Cloud. The code is provided on Github and archived on Zenodo (Hut, 2018).

Figure 5 shows how the data moves from the Particle Electron, via the Particle Cloud service unto a personal webserver. A webhook running on the Particle Cloud service parses the messages from the Particle Electron and posts it to the 'receiveData.php' file on the server. This file first stores the raw data in a dataBase, as a backup measure. Than it parses the message into the individual GPS measurements and stores these in the dataBase labelled 'Processed Data'. Users can look at this data in two ways. By opening the file dataDump.php in a browser a csv dump from the dataBase is generated. This allows researchers to do analyses on the measurements after the field campaign. When realTimeMap.html is opened a single device can be selected from a drop-down box. Once selected a map with the latest measurements from that device is shown. This allows researchers to track the devices in real time. All source code that runs on the personal server, including instructions how to install the Particle webhook, is provided on github and archived on Zenodo (Hut, 2018).

\section{3 power consumption}

115 A critical design criteria for our devices is that they should be able to run continuously on the combination of solar panel and battery. This is not an uncommon constrain for equipment in geoscientific applications. The logging of battery level is an additional feature build into the software running on the Particle Electron. We implemented a deep sleep cycle, where the MCU goes into a deep sleep mode which uses less than 200 micro amperes in between taking measurements from the GPS mod- 
ule. In deep sleep, the GPS unit remains active because it can take up to 20 minutes to achieve a fix on the GPS satellites, a process that takes considerable more power than maintaining this fix.

Testing in the field showed that the device functions for at least two full days on a $1200 \mathrm{mAh}$ battery. The addition of the chosen solar panel extends this to about 4 days, depending on local solar conditions.

\section{4 phyiscal construction}

The Particle Asset Tracker comes delivered with a water proof enclosure. All electrical components except the solar panel fit inside this enclosure. A small hole was drilled in the enclosure to feed the connections from the solar panel to the Particle Electron. The hole, and the connectors on the solar panel, were sealed with two component epoxy to make them waterproof. This was chosen over more conventional closures such as hot-glue because of its excellent thermal properties: once hardened it has negligible expansion when exposed to temperature differences.

We designed, based on local available material a wooden plate with Styrofoam and 5 anchors to give it drag. In our condition, with low wind conditions this design worked fine, but of course design of the floaters need to be tailor made based on local conditions and scientific questions.

\section{3 fieldwork: test in Ayeyarwady}

Experiments were conducted both upstream of the confluence in the Ayeyarwady as well as at the Chindwin-Ayeyarwady confluence. The Ayeyarwady river system is a very dynamic and naturally, unregulated, river and one of the largest rivers in the world and a vital vein for Myanmar (SOBA, 2018). It not only is one of the main transport corridors, it is also the source of fish, irrigation and drinking water and an important aquatic ecosystem (SOBA, 2018). It is a shallow dynamic river system, typically a depth range of 1-10 m only whereas it has a width of several $100 \mathrm{~m}$. The migrating sandbanks, especially upstream and around the confluence with the Chindwin, are a very pressing problem for river management. Insight in hydraulic behaviour of the river is therefore a continuous demand. First of all, groups of five drifters were release at the same time in a line across the river. This allowed to quantify the cross-sectional variation in surface water flow velocity. They were followed, both online and with a trailing boat. For logistical reasons, when all drifters became too dispersed, they were fished out and brought together to restart the experiment. If a single drifter started significantly lacking behind with respect to the rest of the group, it was fished out of the water.

The upstream Ayeyarwady experiment was executed with a single group of five drifters. The confluence experiment was executed with two groups of five drifters upstream of the ChindwinAyeyarwady confluence, one in each river. The drifters were tracked along the confluence. At the 
end of the experiment, drifters were collected and data was read out both from the SD-cards of the OpenLog as well as from the website to which the data had been uploaded.

The raw data collected from the sensors are plotted on a map of the experiment area, see Figure 5. The different flow paths that the sensors followed can clearly be identified. These data being available online directly allowed the measurement team to identify trackers that had beached, or were stuck in near shore eddies. The full raw dataset is provided as supplementary material to this article. This raw data will be used in follow up research. Figure 5 furthermore shows a closeup of the upstream Ayeyarwady experimental results. The location of the drifters clearly shows the different flow paths in the river. Unfortunately, we do spot some gaps in the data. These are caused by lack of cell coverage. In these instances, the Particle Electron took very long in trying to connect, before deciding the switch to offline leading to a loss of data. Future versions of the software code running on the Particle Electron should switch sooner to offline when cell coverage drops to prevent loss of data. Finally, although clearly labeled as experimental equipment, some sensors were picked out of the water by citizens. This was easily identified in the online visualization (ie. sensors moving upstream) and allowed the research team to chase and recover those sensors. These instances were manually removed from the dataset.

\section{4 discussion}

The intention of this research was to test if currently available plug and play sensors and communication solutions are such that a typical member of the geoscientific community can use them to build their own measurement equipment suited to the specifics of the measurement campaign. Our experience shows that many geoscientifically trained persons should be able to connect the different pieces together and build the basic drifters. However, to have the entire setup operate at the required low power levels requires changing the standard programs that run on the device. The level of understanding of the electronic functioning of the device is not something that in general can be expected from a typical geoscientist, even one with extensive experience in using various types of electronic measurement equipment. Furthermore, developing the online back-end that visualizes the location of the GPS drifters in real time required back up from colleagues (see acknowledgements) with web-development expertise. Besides the design of the drifter, also the construction of the trackers in Myanmar required creativity of using local available materials as well as the expertise of the design to be able to fine-tune and debug the tracker in the field. In our case, the code was changed such that if no cell signal was found, the logger would check again in five, instead of one, minutes, to minimize the data gaps (See above). Also, revitalising a drowned tracker (due to collision with a commercial shipping vessel) needed engineering expertise. These type of experiences learned us that the real-world application of self-designed Open Hardware equipment is not that straightforward as sometimes envisioned. Here we advocate that multi-disciplinary collaboration is still required when 
using low-cost self designed equipment. Making the design more robust will come against higher expenses.

Being able to trace the low-cost GPS drifters in real time proved invaluable during the fieldwork. Although the devices were clearly labelled as research equipment, some were picked up by curious locals. This was immediately visible online, e.g. because the sensors started moving upstream or accelerated to unnatural flow velocities. The real time visualization allowed us to recover valuable equipment, which makes this GPS technology also practical as GeoChasing option.

\section{Conclusions}

We presented a low cost GPS drifter that communicates its data in real time over the cellular network. We presented our design such that fellow geoscientist can (re)build or modify our GPS drifters using this article. Our device was tested in an extensive fieldwork in the Chindwin and Ayeyarwady rivers in Myanmar. During development we tested if such a device can be completely developed by a geoscientist without electrical engineering background from available plug and play components. Although creating an online logging GPS drifter turned out to be do-able without electrical engineering background, it required considerable understanding of embedded programming to develop a version that was low power enough to be useful in our fieldwork environment. It would be extremely useful for the geoscientific community if low power versions of the electronics used in this research were available "out of the box". Several companies are building lower power logging equipment for geoscientists Wickert (2014), but these are usually not yet usable without having to program them first.

The online environment that visualizes the field work data in real time as it comes in was developed by the main author and hosted on his personal website. It would be a great asset to geoscientists doing fieldwork if there was a platform that both stored and visualizes real time data coming in. Part of this functionality is build in the CUAHSI Hydroshare project Tarboton et al. (2014), although this focuses more on storing data for prosperity than on real time checking of data as it comes in.

The advance of plug and play electronics has opened the opportunity for geoscientists to patch together the functionality they need in a sensor-logger-communication device. We showed that for

215 a GPS drifter with cellular connectivity and local storage this is indeed possible. However, getting plug and play devices to be low power enough for fieldwork condition still requires considerable electrical engineering knowledge. We hope that this research provides a step in the right direction to help geoscientists use our GPS drifters in particular and plug and play devices in general and encourage the companies producing these devices to bring low power versions to market that work out of the box. 
Acknowledgements. The authors would like to thank MSc and PhD students from YTU and MMU (Yangon, Myanmar), MTU (Mandalay, Myanmar) and TUDelft (The Netherlands), for their assistance in the field. Futhermore we like to thank MSc student Rick Hagenaars for helping developing the online back-end, proving the value of having students with a diverse background and skill set in your team / community.

Finally, the authors would like to thank VPDelta for their support, and in particular the Partner voor Water project: "Leapfrogging Delta Management in Myanmar - Showcase smart information solutions in the Ayeyawady Delta" and NICHE-NMR-250 "Capacity Development on Integrated Water Resources Management in Myanmar"

\section{Author contribution}

230 Author contributions are listed using the CASRAI Credit system Allen et al. (2014)

- Rolf Hut: Conceptualization, Methodology, Investigation, Resources, Software, Validation, Visualization, Writing - original draft, Writing - review and editing.

- Thanda Thatoe Nwe Win: Methodology, Investigation, Project administration, Writing - original draft (section fieldwork: test in Ayeyarwady).

- Thom Bogaard: Conceptualization, Investigation, Resources, Supervision, Writing - review and editing. 


\section{References}

Allen, L., Scott, J., Brand, A., Hlava, M., and Altman, M.: Publishing: Credit Where Credit Is Due, Nature News, 508, 312, doi:10.1038/508312a, 2014.

Arduino: Arduino - Home, https://www.arduino.cc/, 2018.

Banzi, M. and Shiloh, M.: Getting Started with Arduino: The Open Source Electronics Prototyping Platform, Maker Media, Inc., 2014.

Cain, P. W. and Cross, M. D.: An Open-Source Hardware GPS Data Logger for Wildlife Radio-Telemetry Studies: A Case Study Using Eastern Box Turtles, HardwareX, 3, 82-90, doi:10.1016/j.ohx.2018.02.002, 2018.

Chris, A.: Makers: The New Industrial Revolution, New York: Crown Business, 2012.

Costanzo, A.: An Arduino Based System Provided with GPS/GPRS Shield for Real Time Monitoring of Traffic Flows, in: 2013 7th International Conference on Application of Information and Communication Technologies, pp. 1-5, doi:10.1109/ICAICT.2013.6722710, 2013.

Daniel K., F. and Peter J., G.: Open-Source Hardware Is a Low-Cost Alternative for Scientific Instrumentation and Research, Modern Instrumentation, 2012, doi:10.4236/mi.2012.12002, 2012.

Foundation, R. P.: Raspberry Pi, https://www.raspberrypi.org, 2018.

Hut, R.: RolfHut/Low-Power-GPS-Trackers-with-GSM-Modems.-Firmware-and-Online-Backend v1.0, Zenodo, doi:10.5281/zenodo.1485899, 2018.

Lockridge, G., Dzwonkowski, B., Nelson, R., and Powers, S.: Development of a Low-Cost Arduino-Based Sonde for Coastal Applications, Sensors, 16, 528, doi:10.3390/s16040528, 2016.

Network, F. S.: Floating Sensor Network, http://float.berkeley.edu/, 2012.

Particle: Particle Build, https://build.particle.io/build/new, 2018.

Pham, H. D., Drieberg, M., and Nguyen, C. C.: Development of Vehicle Tracking System Using GPS and GSM Modem, in: 2013 IEEE Conference on Open Systems (ICOS), pp. 89-94, doi:10.1109/ICOS.2013.6735054, 2013.

SOBA: The Ayeyarwady State of the Basin Assessment (SOBA), https://www.myanmarwaterportal.com/news/663-the-ayeyarwady-state-of-the-basin-assessment-soba.html, 2018.

Tarboton, D. G., Idaszak, R., Horsburgh, J. S., Heard, J., Ames, D., Goodall, J. L., Band, L., Merwade, V., Couch, A., Arrigo, J., et al.: HydroShare: Advancing Collaboration through Hydrologic Data and Model Sharing, 2014.

Tinka, A., Strub, I., Wu, Q., and Bayen, A. M.: Quadratic Programming Based Data Assimilation with Passive Drifting Sensors for Shallow Water Flows, International Journal of Control, 83, 1686-1700, doi:10.1080/00207179.2010.489621, 2010.

Tinka, A., Rafiee, M., and Bayen, A. M.: Floating Sensor Networks for River Studies, IEEE Systems Journal, 7, 36-49, doi:10.1109/JSYST.2012.2204914, 2013.

Trevathan, J., Johnstone, R., Chiffings, T., Atkinson, I., Bergmann, N., Read, W., Theiss, S., Myers, T., and Stevens, T.: SEMAT - The Next Generation of Inexpensive Marine Environmental Monitoring and Measurement Systems, Sensors, 12, 9711-9748, doi:10.3390/s120709711, 2012. 


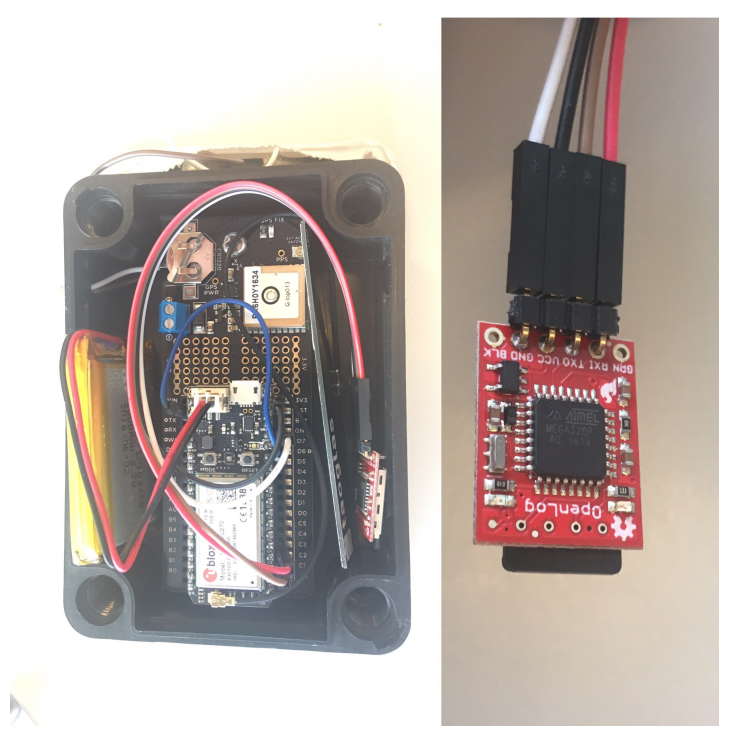

Figure 1. Photo of the electronics components. The left photo identifies the Asset Tracker, Electron and OpenLog. The right close ups show the OpenLog in detail.

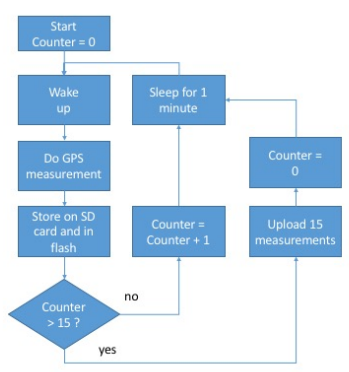

Figure 2. a Flowchart of the software that runs on the Particle Electron. The full code is available on Github (Hut, 2018). In situation where limited power is not an issue, this software can be greatly simplified by removing the part where the Electron goes into power saving deep sleep mode. For our case study, however, low power usage was essential to a successful field campaign. the Ecological Society of America, 95, 166-176, doi:10.1890/0012-9623-95.2.68, 2014. 


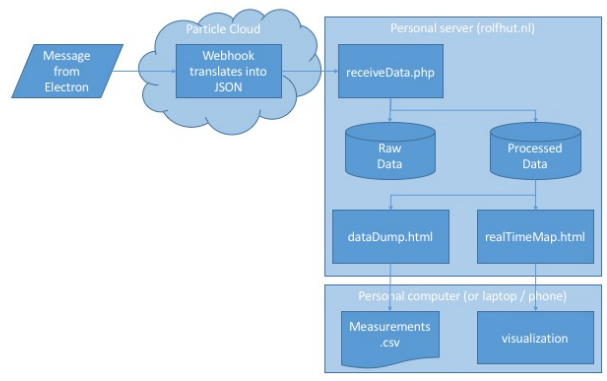

Figure 3. The flow of data once it leaves the Particle Electron. The Electron sends the measurements as a comma seperated message to the Particle Cloud. A webhook running on the Particle Cloud parses the raw message into a JSON message that is posted to a php file on the personal server of the main author. This file adds the data to two databases: one to store all raw messages, one that parses the message into the individual measurements. Two html files can be called by users. DataDump.html transforms the database in a comma seperated file for downloading. realTimeMap.html shows the last known locations of the drifters for real time localization during the field campaign. All software is available on github (Hut, 2018)

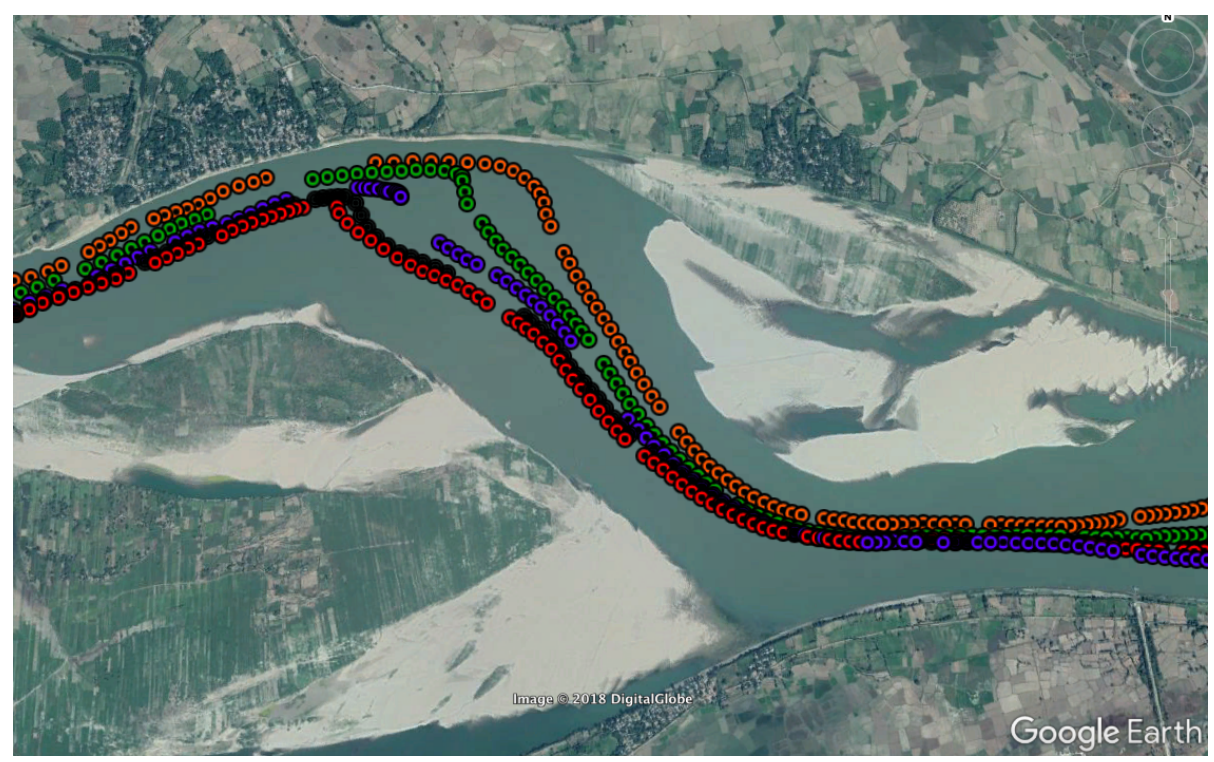

Figure 4. The location measurements for the different floaters (different colors) in the Irrawady river near Ngazun. (G)oogle Earth 2019 


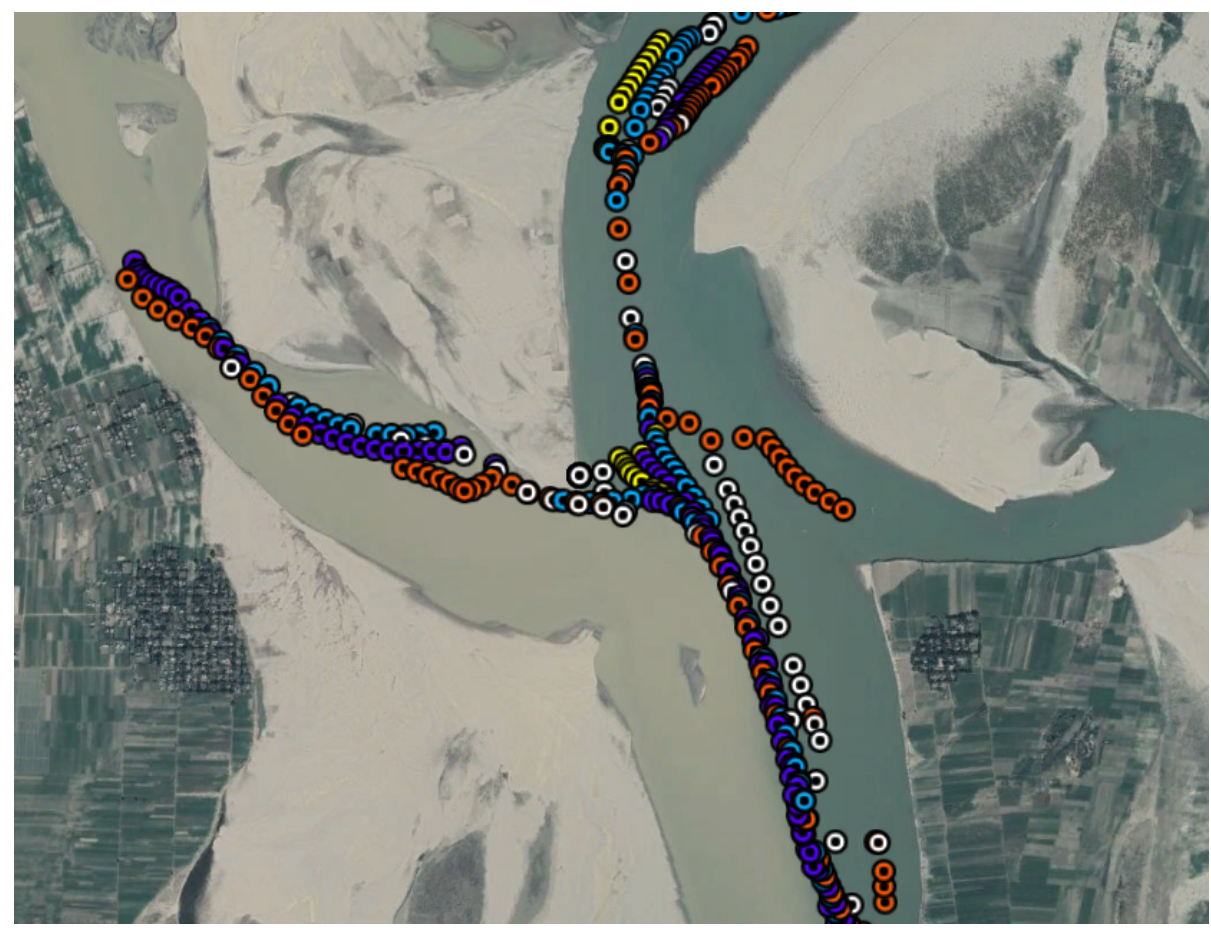

Figure 5. The location measurements for the different floaters (different colors) at the confluence of the Chindwin and Irrawaddy rivers. OGoogle Earth 2019

Table 1. Price and online location of information on off the shelf electronical components used to build the Low power GPS drifters with local storage and GSM modem.

\begin{tabular}{lll}
\hline Name & Price & Online location \\
\hline Particle Asset Tracker & $\$ 109$ & https://www.particle.io/products/hardware/asset-tracker \\
Particle Electron & included with Asset Tracker & https://www.particle.io/products/hardware/electron-cellular-dev-kit \\
SparkFun OpenLog & $\$ 14.95$ & https://www.sparkfun.com/products/13712 \\
Conrad Solar Panel & $€ 14.95$ & https://www.conrad.nl/nl/polykristallijn-zonnepaneel-09-wp-6-v-110454.html \\
\hline
\end{tabular}


https://doi.org/10.5194/gi-2019-27

Preprint. Discussion started: 7 October 2019

(c) Author(s) 2019. CC BY 4.0 License.

Table 2. Electrical connections between the Particle Asset Tracker and the other components. Note that the connection between GND and OpenLog BLK can be made by connecting the OpenLog pins BLK and GND, saving the need for one wire. The connection between pin D6 and GND is to force the GPS unit on. See figure 5 for an image of the physical setup.

\begin{tabular}{|l|l|}
\hline pin on AssetTracker & connected to \\
\hline \begin{tabular}{l|l|} 
power terminal - \\
power terminal +
\end{tabular} & $\begin{array}{l}\text { solar panel - } \\
\text { solar panel + }\end{array}$ \\
\hline C2 & OpenLog TX0 \\
C3 & OpenLog RX1 \\
GND & OpenLog GND \\
GND & OpenLog BLK \\
3V3 & OpenLog VCC \\
\hline D6 & Asset Tracker GND \\
\hline
\end{tabular}

